\title{
Dynamics of Mitral Complex Geometry and Functional Mitral Regurgitation During Heart Failure Treatment -Real-time Three-dimensional Echocardiographic Study-
}

\author{
Nozomi Watanabe, MD*, Yasuo Ogasawara, PhD**, Yasuko Yamaura, MD* \\ Katsunori Yamamoto, RDCS*, Nozomi Wada, MD*, Noriko Okahashi, MD*, \\ Takahiro Kawamoto, MD*, Eiji Toyota, MD* and Kiyoshi Yoshida, MD* \\ *Department of Cardiology \\ **Department of Medical Engineering and Systems of Cardiology, Kawasaki Medical School, \\ Kurashiki, Japan
}

\begin{abstract}
Background. Although mitral complex geometry alteration has been speculated as a major mechanism for the reduction in mitral regurgitation (MR) after heart failure treatment, it has been difficult to recognize the mechanistic changes of the mitral apparatus because of the complex morphology and anatomical position. We investigated the contributions of the dynamic changes in mitral valve geometry with papillary muscle (PM) alignment to the reduction of functional MR after intensive heart failure treatment.

Methods. Two dimensional/three-dimensional (2D/3D) echocardiography was performed in 10 patients with decompensated heart failure and functional MR before and after intensive therapy. We used novel software to analyze the volumetric images recorded by real-time 3D echocardiography. Reconstructed 3D images showed both leaflets and annulus configuration in relation to PM position. We measured maximum tenting length (max-Tent-L); mean tenting length (mean-Tent-L) and tenting volume (Tent-V); distances from anterior annulus to anterolateral PM (A-tethering length) and posteromedial PM (P-tethering length); the distance between two PM (interpapillary distance); and the angle made by the anterolateral PM, anterior annulus, and posteromedial PM (interpapillary angle).

Results. MR decreased after intensive treatment in all patients $(p<0.0001)$. Leaflet tenting decreased significantly after therapy (max-Tent-L, $16.8 \pm 2.5$ vs. $13.3 \pm 2.0 \mathrm{~mm}, p<0.001$; mean-Tent-L, $9.5 \pm 2.1$ vs. $7.3 \pm 1.3 \mathrm{~mm}, p<0.001$; Tent-V, $10.3 \pm 2.8$ vs. $6.2 \pm 1.6 \mathrm{ml}, p=0.0002)$. P-tethering length shortened ( $46.9 \pm 5.1$ vs. $38.6 \pm 4.9 \mathrm{~mm}, \mathrm{p}<0.001)$. Interpapillary distance $(25.8 \pm 5.6$ vs. $17.2 \pm 4.0 \mathrm{~mm}, \mathrm{p}<0.001)$ and interpapillary angle $(32.0 \pm 7.3$ vs. $23.8 \pm 7.5$ degree, $p<0.001)$ decreased after treatment.

Conclusions. Dynamic changes in PM position during heart failure treatment resulted in the reduction of mitral valve tenting, which improved functional MR.

(J Echocardiogr 2006; 4: 51-58)
\end{abstract}

Key words: functional mitral regurgitation, mitral complex, heart failure

\section{Introduction}

Functional mitral regurgitation (MR) is frequently observed in patients with congestive heart failure (CHF) [1]. It has been well established that the degree

Received May 10, 2006; revision received June 12, 2006; accepted June 17, 2006

Address for correspondence: Nozomi Watanabe, MD, PhD, FACC

Department of Cardiology, Kawasaki Medical School,

577 Matsushima, Kurashiki 701-0192, Japan.

Telephone: +81-86-462-1111

Fax: +81-86-462-1199

E-mail: non@med.kawasaki-m.ac.jp

C. 2006 Japanese Society of Echocardiography of MR dramatically decreases after intensive vasodilator and diuretic therapy or angiotensin-converting enzyme (ACE) inhibitor therapy for decompensated heart failure, and reduced MR volume contributes to the improvement of cardiac output [2-8]. In functional MR, the shift in papillary muscle position due to ventricular dilatation is assumed to lead to tenting of the mitral valve, which causes an ineffective leaflet coaptation [9-14]. Although previous studies have speculated alterations in the mitral apparatus geometry as the major cause of reduction in the regurgitant orifice during heart failure treatment $[2,3,15,16]$, it has been difficult to recognize the mechanistic changes of the 
mitral annulus, leaflets, and papillary muscles because of their complex morphologies and anatomical positions. Recently we developed a novel software system which allows us three-dimensional (3D) visualization and quantitation of mitral leaflets and annulus geometry from real-time 3D echocardiographic images [1720]. In this study, we investigated the contributions of dynamic changes in the mitral leaflets and annulus geometry with papillary muscle alignment to the reduction of functional MR after intensive treatment for the patients with decompensated heart failure with the use of transthoracic real-time 3D echocardiography.

\section{Methods}

\section{Patients.}

We studied ten patients with $\mathrm{CHF}$ and functional MR (regurgitant orifice area $[\mathrm{ROA}]>0.2 \mathrm{~cm}^{2}$ ), who were classified as Class 3 or 4 of the New York Heart Association (8 male, 2 female, $75 \pm 9$ years). CHF was secondary to coronary artery disease in 5 patients and idiopathic dilated cardiomyopathy in 5 patients. Inclusion criteria were [1] structurally normal mitral valve; [2] technically adequate color flow Doppler image for proximal isovelocity surface area (PISA); [3] technically adequate real-time 3D echocardiographic images of the left ventricle (LV), papillary muscles, the mitral annulus, and leaflets to allow 3D geometric analysis, and [4] normal sinus rhythm. Exclusion criteria were 1) acute myocardial infarction; 2) structural mitral valve or subvalvular lesions, such as mitral valve prolapse or rheumatic disease; 3 ) other cardiac disease such as organic valvular, pericardial, congenital, or infiltrative heart disease. Pharmacologic therapy included intravenous sodium nitroprusside and intravenous furosemide. Low dose dobutamine was employed in 6 patients. All patients were prescribed ACE inhibitors. All participants gave written, informed consent to the study protocol, which was approved by the Committee for the Protection of Human Subjects in Research at Kawasaki Medical School.

\section{Echocardiographic protocol.}

All the echocardiographic exams were performed utilizing a SONOS $7500^{\circledR}$ (Philips Ultrasound, Bothell, Washington) with a S3 probe for 2D images and an X4 probe for real-time 3D images. Initial exams were performed prior to the initiation of intensive therapy for $\mathrm{CHF}$, and the patients were reexamined when the patients were receiving a stable dose of oral medications.
$<$ Two-dimensional Echocardiographic Study>

All subjects underwent a standard 2D echocardiographic examination at the beginning and conclusion of the intensive vasodilator and diuretic treatments. MR was evaluated by color Doppler echocardiography. The degree of MR was quantified by ROA using the PISA method. When MR degree was none or trivial (as assessed using color Doppler), MR volume and ROA were assumed to be null. Doppler pulsed-wave tracings of LV inflow were obtained from apical views. The ratio of maximal velocities of $\mathrm{E}$ wave to $\mathrm{A}$ wave (E/A) and $\mathrm{E}$ wave deceleration time were measured. Pulmonary arterial pressure was estimated by the equation $4 \times \mathrm{V}^{2}+10 \mathrm{mmHg}$, while $\mathrm{V}(\mathrm{m})$ was the tricuspid regurgitant velocity measured via continuous Doppler method.

$<$ Three-dimensional Echocardiographic Study>

Volumetric Image Acquisition. Utilizing real-time 3D echocardiographic system, we obtained transthoracic volumetric images (full volume mode) with the apical view in all subjects. The volumetric frame rate was 11 to 18 frames/second, with an imaging depth of 14 to $18 \mathrm{~cm}$. Prior to the acquisition of the full volume image, the transducer position was adjusted to the apex in the bi-plane mode. All volumetric images were digitally stored on compact disk and transferred into a personal computer for offline analysis.

LV Volume Measurement. In each exam, LV end-diastolic volume (LVEDV) and end-systolic volume (LVESV) were measured utilizing 3D quantitation software $\mathrm{QLAB}^{\circledR}$ (Philips Ultrasound, Bothell, Washington). Ejection fraction $(\mathrm{EF} \%)$ was calculated as $100 \times(\mathrm{EDV}-$ ESV)/EDV.

Quantitation of Mitral Valve Tenting. We used a novel 3D computer software REAL VIEW ${ }^{\circledR}$ (Y.D., Ltd, Osaka, Japan) to analyze the volumetric image. As per the previous studies [17-20], using a cross-sectional plane of the mitral annulus, we defined the center of the annulus in the volumetric image in order to set the axis through the transducer and the center of the annulus. We also determined the anterior-posterior axis and the commissure-commisure axis in the volumetric image. The 3D data was then automatically cropped into 18 radial planes spaced 10 degrees apart. The mitral annulus in each cropped plane in mid-systole were marked manually. Mitral leaflets were semi-automatically traced. The tips of the anterolateral and posteromedial papillary muscles were obtained on the cropped planes and were marked. From these data, 3D images 
of the mitral leaflets and annulus were reconstructed. Positions of the tips of each papillary muscle were placed in the 3D data (Figure 1). The 3D images show the configuration of the leaflet curvature using surface colorations and curved annulus ring, with an alignment of papillary muscle tips (Figure 2). This image can be rotated and observed from any direction. To assess the geometric changes of the mitral annulus, the annular area and annular circumference were directly analyzed from the $3 \mathrm{D}$ images. The maximum tenting length, mean tenting length, and tenting volume were measured for the evaluation of mitral leaflet geometry. The maximum tenting length was defined as the distance from the annular plane to the most tethered leaflet site in the 3D data. The mean tenting length was defined as the average distance from the annular plane to the tethered leaflet into the LV. The tenting volume was calculated as the volume enclosed between the annular plane and mitral leaflets. To observe the shift in papillary muscle position during therapy, we measured the distances from anterior mitral annulus to each papillary muscle (tethering lengths), the distance between two tips of papillary muscles (interpapillary distance), and the angle that is made of the two lines connecting anterior mitral annulus to each papillary muscle (interpapillary angle). The anterior mitral annulus, which is relatively fixed, was defined as a medial junction of aortic and mitral annuli.

\section{Statistical Analysis.}

Data are expressed as mean \pm SD. Group comparisons used Student's $t$ test. A value of $\mathrm{p}<0.05$ was considered statistically significant.

\section{Results}

Between initiation and conclusion of therapy, there were significant reductions in body weight in all patients. Initial New York Heart Association classifica-
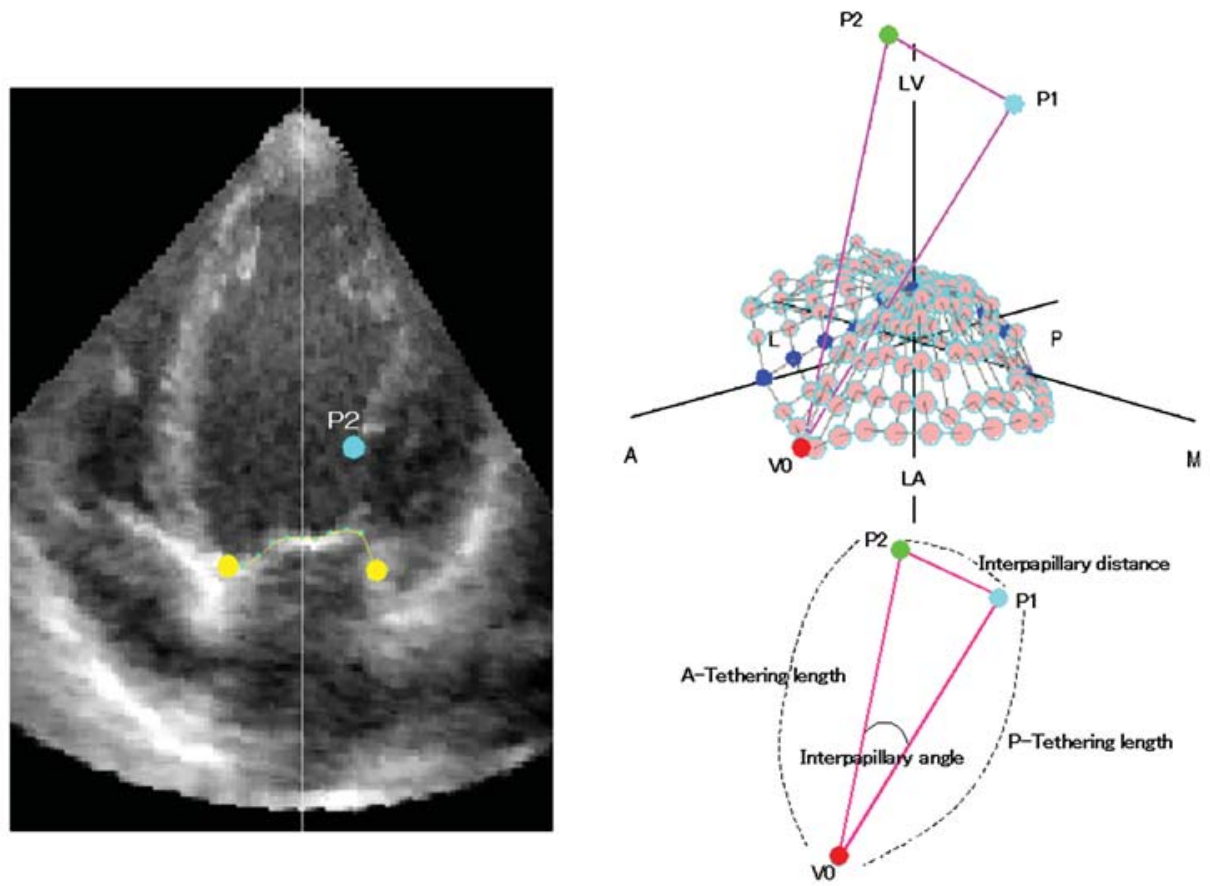

Fig. 1. 3D data was automatically cropped into 18 radial planes spaced 10 degrees apart. The mitral annulus and leaflets were traced in each cropped plane. Tips of the posteromedial papillary muscle and anterolateral papillary muscle were obtained and marked. From this data, 3D images of the mitral leaflets and annulus were reconstructed with position of two papillary tips. Measurements of interpapillary distance, interpapillary angle, and tethering lengths between anterior mitral annulus and anterolateral papillary muscle (A-tethering length) and the posteromedial papillary muscle (P-tethering length) are schematically shown.

( $A$, anterior side; $P$, posterior side; $M$, medial side; $L$, lateral side; V0, anterior mitral annulus; P1, posteromedial papillary muscle; P2, anterolateral papillary muscle) 
tion improved from $3.8 \pm 0.4$ to $2.8 \pm 0.4(\mathrm{p}<0.0001)$. The mean period between the initial and follow-up exams was $16 \pm 5.9$ days.

\section{Echocardiographic and Doppler paramater}

In Table 1, measurements of the LV chamber and Doppler parameters are summarized. LV volume and EF improved, and the estimated pulmonary arterial pressure reduced significantly with therapy. The LV inflow pattern demonstrated a decrease in E/A and an increase in $\mathrm{E}$ wave deceleration time. The MR volume and ROA decreased after therapy in all patients.

Three-dimensional Echocardiographic Analysis of the Mitral Complex

Geometric changes of mitral annulus and leaflets with alignment of papillary muscles are shown in Table 2.

\section{Mitral annular and leaflet geometry.}

Figure 2 shows the 3D images created by REAL
VIEW. In the initial exam, mountain-shaped leaflet bulging of the mitral leaflets, which were tethered into the LV, was observed (mitral valve tenting). The calculated annular area and annular circumference were significantly reduced after $\mathrm{CHF}$. The calculated maximum tenting length, mean tenting length, and tenting volume also decreased significantly after therapy.

Anatomical position of papillary muscles and mitral apparatus

Changes in the mitral apparatus geometry with heart failure treatment are shown in Figure 2. The papillary muscle position shifted after therapy. The tethering length from the anterior mitral annulus to the posteromedial papillary muscle was significantly shortened after therapy. The tethering length from the anterior mitral annulus to the anterolateral papillary muscle was also shortened but not significant. The distance between the papillary muscles significantly decreased

Before Therapy

a.

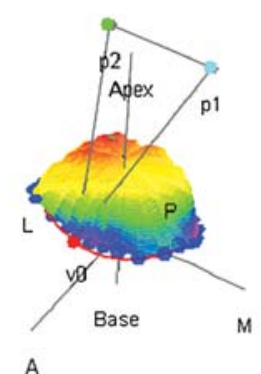

A b.

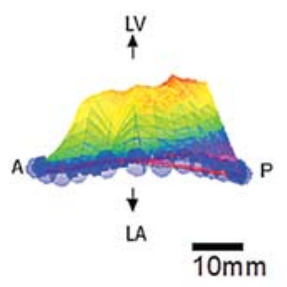

c.

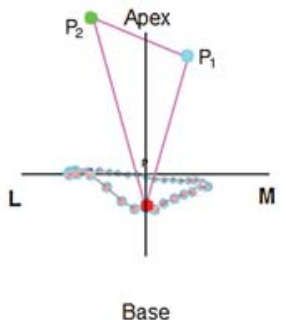

After Therapy

a.

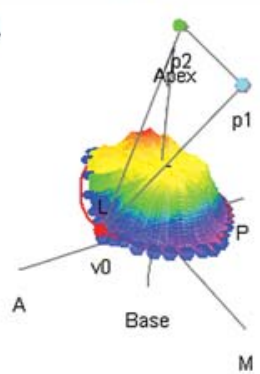

b.

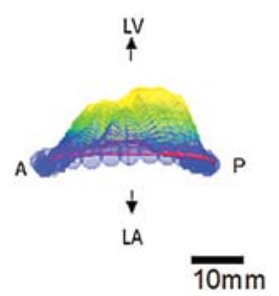

c.

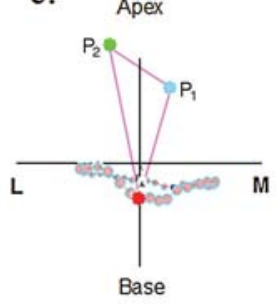

Fig. 2. Upper panel shows $3 D$ images before heart failure treatment, and lower panel, after heart failure treatment.

a. 3D images of the mitral leaflets and annulus in combination with papillary muscle (PM) alignment.

b. Horizontal view of the 3D images of the mitral leaflets and annulus. Leaflet tenting is significantly reduced status post CHF therapy.

c. 3D images of the mitral annulus in combination with PM alignment. Anterolateral PM and posteromedial PM becomes closer after the treatment.

( $A$, anterior side; $\mathrm{P}$, posterior side; $\mathrm{M}$, medial side; L, lateral side; V0, anterior mitral annulus; P1, posteromedial papillary muscle; P2, anterolateral papillary muscle; LV, left ventricle; LA, left atrium) 
Table 1. Echocardiographic Parameter

\begin{tabular}{lccc}
\hline & Before therapy & After therapy & $p$ value \\
\hline MR & & & \\
ROA, cm ${ }^{2}$ & $0.38 \pm 0.1$ & $0.11 \pm 0.08$ & $<0.0001$ \\
$\quad$ Regurgitant volume, ml & $52.1 \pm 11.6$ & $16.6 \pm 13.6$ & $<0.0001$ \\
LV chamber & & & \\
EDV, ml & $187.8 \pm 46.2$ & $152.0 \pm 45.6$ & $<0.001$ \\
ESV, ml & $150.4 \pm 42.8$ & $110.9 \pm 43.3$ & $<0.0001$ \\
EF, $\%$ & $20.2 \pm 6.7$ & $28.6 \pm 11.1$ & 0.025 \\
LV inflow & & & $<0.0001$ \\
E/A & $2.3 \pm 0.6$ & $1.1 \pm 0.4$ & $<0.001$ \\
DcT, msec & $118.3 \pm 12.9$ & $207.3 \pm 61.6$ & $<0.0001$ \\
PAP, mmHg & $54.5 \pm 5.9$ & $37.9 \pm 4.4$ & \\
\hline
\end{tabular}

ROA indicates regurgitant orifice area; EDV, LV end-diastolic volume; ESV, LV end-systolic volume; EF, ejection fraction; E, E wave velocity; A, A wave velocity; DcT, Deceleration time of E wave, and PAP, estimated pulmonary arterial pressure

Table 2. Measurements of Mitral Complex

\begin{tabular}{lrrr}
\hline & Before therapy & After therapy & $p$ value \\
\hline Mitral Annulus & & & \\
$\quad$ Circumference, cm & $13.0 \pm 1.0$ & $11.6 \pm 1.0$ & $<0.001$ \\
$\quad$ Area, cm ${ }^{2}$ & $13.2 \pm 1.9$ & $10.5 \pm 1.7$ & $<0.001$ \\
Mitral Leaflet & & & \\
$\quad$ Tenting volume, ml & $10.3 \pm 2.8$ & $6.2 \pm 1.6$ & $<0.001$ \\
$\quad$ Maximum tenting length, mm & $16.8 \pm 2.5$ & $13.3 \pm 2.0$ & $<0.001$ \\
Mean tenting length, mm & $9.5 \pm 2.1$ & $7.3 \pm 1.3$ & $<0.01$ \\
Papillary Muscle & & & \\
$\quad$ Tethering length (PM-A), mm & $46.2 \pm 7.2$ & $43.1 \pm 4.9$ & 0.052 \\
$\quad$ Tethering length (PM-P), mm & $46.9 \pm 5.1$ & $38.6 \pm 4.9$ & $<0.001$ \\
$\quad$ Interpapillary distance, mm & $25.8 \pm 5.6$ & $17.2 \pm 4.0$ & $<0.001$ \\
$\quad$ Interpapillary angle, degree & $32.0 \pm 7.3$ & $23.8 \pm 7.5$ & $<0.001$ \\
\hline
\end{tabular}

PM-A indicates anterolateral papillary muscle; PM-P, posteromedial papillary muscle.

with CHF therapy, and, compared to the initial angle, the interpapillary angle was significantly smaller after therapy.

\section{Discussion}

In the present study, we demonstrated dynamic changes in the mitral complex geometry during intensive treatment in decompensated heart failure with functional MR. MR improved after therapy with reduction of LV size. With an improvement in CHF condition, mitral valve tenting volume and tenting length decreased with a reduction in annular size. Positions of papillary muscles shifted after therapy, which should have led to the improvement of mitral tenting, and then, MR volume.

Our novel software system made it possible to visualize and quantify the complex geometry of the mitral annulus and leaflets with the use of transthoracic realtime 3D echocardiography in humans [17-20]. The newest version of the software that was used in the present study allows us to appreciate the anatomical position of papillary muscles, which is believed to be the most important factor in the mechanisms of functional MR. 
Functional MR is a common finding in patients with $\mathrm{CHF}$; the presence of functional MR is known to contribute to the progression of ventricular dysfunction and worsens the prognosis in patients with both ischemic/nonischemic heart failure [1, 16, 21-24]. Dynamic reduction of functional MR after vasodilator and diuretic therapy or ACE inhibitor therapy for heart failure has been reported previously [3, 5-8]. The presence of significant MR may also predict a salutary response to intensive therapy $[25,26]$. The mechanisms of this dynamic change of functional MR have been investigated in both anatomic and hemodynamic views for years, and reduction in the annular size, decrease in afterload, changes in transmitral pressure gradients had been postulated as mechanisms in this dynamics of MR [2, 4, 8, 27-33]. Several studies assumed that a decrease in ROA was responsible for the reduction in MR volume [28-30]; Rosario et al. has demonstrated in the clinical setting that a reduction in the dynamic MR of heart failure after intensive therapy is related to a reduction in the ROA, but not through a change in the transmitral pressure gradients [15]. They suggested that a reduction in ventricular volume would most probably be the mechanism responsible for the decrease in regurgitant orifice with facilitating mitral valve coaptation by improving the function of the mitral annulus and subannular apparatus. Recent investigations have proven that in functional MR, displacement of the attached papillary muscles tethers the mitral leaflets into the LV and restricts their ability to coapt effectively at the level of the mitral annulus, which may also dilate $[9,10,12-14]$. Precise and comprehensive understanding of the 3D geometric changes of the mitral complex, including mitral leaflets and annulus with papillary muscle position, is needed to determine the roles of the annulus and the subvalvar apparatus in relation to the dynamic MR. However, conventional 2D echocardiography could not provide the complex geometry of the unique configuration of the curved mitral valve and saddle-shaped mitral annulus, especially in combination with papillary muscle position. In the present study, we successfully clarified the dynamic changes of mitral complex geometry after intensive therapy for decompensated heart failure with the use of a newly developed software system and real-time transthoracic 3D echocardiography. To our knowledge, this is the first report to provide $3 \mathrm{D}$ geometry of the mitral leaflets and annulus with papillary muscle alignment in humans.

\section{Quantitation of mitral valve tenting in $3 D$ images.}

In all patients, leaflet tenting was observed clearly with dramatic deformation tethered into the LV, showing a mountain-like configuration. After intensive treatment for congestive heart failure, the degree of leaflet tenting was significantly reduced compared with the initial condition, although the leaflet tenting still remained. Our results support the theory that alteration in the mitral apparatus geometry is the major mechanism for the improvement in MR during heart failure treatment. Observation and quantitation of mitral apparatus in 3D views should be useful in the evaluation of dynamic changes of mitral apparatus geometry in various clinical conditions, such as after surgery for functional MR.

\section{Quantitation of mitral annulus configuration.}

In the present study, we quantitatively demonstrated dynamic change of mitral annulus geometry during heart failure treatment. Mitral annular size was significantly reduced after therapy compared with those measured at the initial exam. Recent investigation using 3D echocardiography has shown that the mitral annulus size links LV dilatation to functional MR [34]. Noninvasive analysis of the mitral annulus geometry would be a great contribution in assessing the annular configurations, especially in conjunction with annuloplasty, which plays a big role in the surgical treatment for functional MR.

Geometric change of papillary muscle alignment in combination with mitral annulus.

Currently, a shift in papillary muscle position due to LV dilation is assumed to lead to mitral valve tenting, which causes an ineffective leaflet coaptation then regurgitation [9-14]. Thus, the anatomical position of papillary muscles should be an important factor in assessing the mechanisms of functional MR. Previously in an animal study, Hung et al. demonstrated 3D analysis of papillary muscle repositioning by transesophageal echocardiographic images through water bath [35]. They showed that the inward shift of papillary muscle position resulted in the reduction of MR. In the present study, we demonstrated the dynamic changes of papillary muscle position noninvasively in the clinical setting, accompanied by the dynamic changes of mitral leaflet geometry. Our novel software system with transthoracic 3D echocardiography allowed us to measure not only tethering lengths but also the interpapillary distance and angle, which can be measured only in 3D datasets. Our data quanti- 
tatively showed the dynamic shift of papillary muscles with the reduction of valve tethering. Interestingly in the present study, shortening of the tethering length was significant with the posteromedial papillary musclebut not significant with the anterolateral papillary muscle. Changes in papillary muscle position may reflect a unique geometric change of remodeled LV. This $3 \mathrm{D}$ quantitation system also provides a unique opportunity to evaluate dynamic changes of the mitral complex geometry through the cardiac cycle independent of the twisting motion of the heart.

Study Limitations.

1) Real-time 3D echocardiography currently available in the clinical setting provides images with lower quality than conventional 2D echocardiography. As the software system used in this study requires identification of mitral annulus, leaflets and papillary muscles for tracing, technically inadequate real-time 3D echocardiographic images are not amenable for analysis. 2) For this study, we enrolled CHF patients with ishemic and nonischemic heart disease. There could be geometric differences in the mitral complex in various types of underlying diseases. Further investigation would be required in this regard. 3) Albeit this method has not been validated, we estimated MR severity in patients with 2 jets by the summation of 2 jets via the PISA method.

\section{Conclusions}

Dynamic changes in mitral complex geometry during heart failure treatment were clearly demonstrated by 3D echocardiography with the use of a newly developed software system. Changes of papillary muscle position during $\mathrm{CHF}$ treatment resulted in the reduction of mitral valve tenting, which resulted in the improvement of functional MR.

\section{References}

1. Robbins JD, Maniar PB, Cotts W, Parker MA, Bonow RO, Gheorghiade M. Prevalence and severity of mitral regurgitation in chronic systolic heart failure. Am J Cardiol 2003; 91: 360-2.

2. Keren G, Bier A, Strom JA, Laniado S, Sonnenblick EH, LeJemtel TH. Dynamics of mitral regurgitation during nitroglycerin therapy: a Doppler echocardiographic study. Am Heart J 1986; 112: 517-25.

3. Weiland DS, Konstam MA, Salem DN, et al. Contribution of reduced mitral regurgitant volume to vasodilator effect in severe left ventricular failure secondary to coronary artery disease or idiopathic dilated cardiomyopathy. Am J
Cardiol 1986; 58: 1046-50.

4. Keren G, Laniado S, Sonnenblick EH, Lejemtel TH. Dynamics of functional mitral regurgitation during dobutamine therapy in patients with severe congestive heart failure: a Doppler echocardiographic study. Am Heart J 1989; 118: 748-54.

5. Varriale P, David W, Chryssos BE. Hemodynamic response to intravenous enalaprilat in patients with severe congestive heart failure and mitral regurgitation. Clin Cardiol 1993; 16: 235-8.

6. Levine TB, Levine AB, Keteyian SJ, Narins B, Lesch M. Reverse remodeling in heart failure with intensification of vasodilator therapy. Clin Cardiol 1997; 20: 697-702.

7. Campos PC, D’Cruz IA, Johnson LS, Malhotra A, Ramanathan KB, Weber KT. Functional valvular incompetence in decompensated heart failure: noninvasive monitoring and response to medical management. Am J Med Sci 2005; 329: 217-21.

8. Stevenson LW, Bellil D, Grover-McKay M, et al. Effects of afterload reduction (diuretics and vasodilators) on left ventricular volume and mitral regurgitation in severe congestive heart failure secondary to ischemic or idiopathic dilated cardiomyopathy. Am J Cardiol 1987; 60: 654-8.

9. Otsuji Y, Handschumacher MD, Schwammenthal E, et al. Insights from three-dimensional echocardiography into the mechanism of functional mitral regurgitation: direct in vivo demonstration of altered leaflet tethering geometry. Circulation 1997; 96: 1999-2008.

10. He S, Fontaine AA, Schwammenthal E, Yoganathan AP, Levine RA. Integrated mechanism for functional mitral regurgitation: leaflet restriction versus coapting force: in vitro studies. Circulation 1997; 96: 1826-34.

11. Yiu SF, Enriquez-Sarano M, Tribouilloy C, Seward JB, Tajik AJ. Determinants of the degree of functional mitral regurgitation in patients with systolic left ventricular dysfunction: A quantitative clinical study. Circulation 2000; 102: $1400-6$.

12. Otsuji Y, Handschumacher MD, Liel-Cohen N, et al. Mechanism of ischemic mitral regurgitation with segmental left ventricular dysfunction: three-dimensional echocardiographic studies in models of acute and chronic progressive regurgitation. J Am Coll Cardiol 2001; 37: $641-8$.

13. Otsuji Y, Kumanohoso T, Yoshifuku S, et al. Isolated annular dilation does not usually cause important functional mitral regurgitation: comparison between patients with lone atrial fibrillation and those with idiopathic or ischemic cardiomyopathy. J Am Coll Cardiol 2002; 39: 1651-6.

14. Levine RA, Hung J, Otsuji Y, et al. Mechanistic insights into functional mitral regurgitation. Curr Cardiol Rep 2002; 4: 125-9. 
15. Rosario LB, Stevenson LW, Solomon SD, Lee RT, Reimold SC. The mechanism of decrease in dynamic mitral regurgitation during heart failure treatment: importance of reduction in the regurgitant orifice size. J Am Coll Cardiol 1998; 32: 1819-24.

16. Bursi F, Enriquez-Sarano M, Nkomo VT, et al. Heart failure and death after myocardial infarction in the community: the emerging role of mitral regurgitation. Circulation 2005; 111: 295-301.

17. Yamaura Y, Watanabe N, Ogasawara Y, et al. Geometrical Demonstration and Three-Dimensional Quantitative Analysis of the Mitral Valve With Real Time ThreeDimensional Echocardiography: Novel Anatomical Image Creation System. J Echocardiogr 2004; 2: 99-104.

18. Watanabe N, Ogasawara Y, Yamaura Y, et al. Quantitation of mitral valve tenting in ischemic mitral regurgitation by transthoracic real-time three-dimensional echocardiography. J Am Coll Cardiol 2005; 45: 763-9.

19. Watanabe N, Ogasawara Y, Yamaura Y, Kawamoto T, Akasaka T, Yoshida K. Geometric deformity of the mitral annulus in patients with ischemic mitral regurgitation: a real-time three-dimensional echocardiographic study. J Heart Valve Dis 2005; 14: 447-52.

20. Watanabe N, Ogasawara Y, Yamaura Y, et al. Mitral annulus flattens in ischemic mitral regurgitation: geometric differences between inferior and anterior myocardial infarction: a real-time 3-dimensional echocardiographic study. Circulation 2005; 112: I458-62.

21. Blondheim DS, Jacobs LE, Kotler MN, Costacurta GA, Parry WR. Dilated cardiomyopathy with mitral regurgitation: decreased survival despite a low frequency of left ventricular thrombus. Am Heart J 1991; 122: 763-71.

22. Koelling TM, Aaronson KD, Cody RJ, Bach DS, Armstrong WF. Prognostic significance of mitral regurgitation and tricuspid regurgitation in patients with left ventricular systolic dysfunction. Am Heart J 2002; 144: 524-9.

23. Tcheng JE, Jackman JD, Jr., Nelson CL, et al. Outcome of patients sustaining acute ischemic mitral regurgitation during myocardial infarction. Ann Intern Med 1992; 117: 18-24.

24. Salukhe TV, Henein MY, Sutton R. Ischemic mitral regurgitation and its related risk after myocardial infarction. Circulation 2005; 111: 254-6.

25. Evangelista-Masip A, Bruguera-Cortada J, SerratSerradell $\mathrm{R}$, et al. Influence of mitral regurgitation on the response to captopril therapy for congestive heart failure caused by idiopathic dilated cardiomyopathy. Am J Cardiol 1992; 69: 373-6.

26. Keren G, Katz S, Strom J, Sonnenblick EH, LeJemtel TH. Dynamic mitral regurgitation. An important determinant of the hemodynamic response to load alterations and inotropic therapy in severe heart failure. Circulation 1989; 80: 306-13.

27. Yoran C, Yellin EL, Becker RM, Gabbay S, Frater RW, Sonnenblick EH. Mechanism of reduction of mitral regurgitation with vasodilator therapy. Am J Cardiol 1979; 43: 773-7.

28. Yoran C, Yellin EL, Becker RM, Gabbay S, Frater RW, Sonnenblick EH. Dynamic aspects of acute mitral regurgitation: effects of ventricular volume, pressure and contractility on the effective regurgitant orifice area. Circulation 1979; 60: 170-6.

29. Yellin EL, Yoran C, Sonnenblick EH, Gabbay S, Frater RW. Dynamic changes in the canine mitral regurgitant orifice area during ventricular ejection. Circ Res 1979; 45: 677-83.

30. Borgenhagen DM, Serur JR, Gorlin R, Adams D, Sonnenblick EH. The effects of left ventricular load and contractility on mitral regurgitant orifice size and flow in the dog. Circulation 1977; 56: 106-13.

31. Boltwood CM, Tei C, Wong M, Shah PM. Quantitative echocardiography of the mitral complex in dilated cardiomyopathy: the mechanism of functional mitral regurgitation. Circulation 1983; 68: 498-508.

32. Hamilton MA, Stevenson LW, Child JS, Moriguchi JD, Woo M. Acute reduction of atrial overload during vasodilator and diuretic therapy in advanced congestive heart failure. Am J Cardiol 1990; 65: 1209-12.

33. Capomolla S, Pozzoli M, Opasich C, et al. Dobutamine and nitroprusside infusion in patients with severe congestive heart failure: hemodynamic improvement by discordant effects on mitral regurgitation, left atrial function, and ventricular function. Am Heart J 1997; 134: 1089-98.

34. Popovic ZB, Martin M, Fukamachi K, et al. Mitral annulus size links ventricular dilatation to functional mitral regurgitation. J Am Soc Echocardiogr 2005; 18: 959-63.

35. Hung J, Guerrero JL, Handschumacher MD, Supple G, Sullivan S, Levine RA. Reverse ventricular remodeling reduces ischemic mitral regurgitation: echo-guided device application in the beating heart. Circulation 2002; 106: 2594-600. 